\title{
Trends in Transplantation
}

\section{Dismal survival following liver transplantation for liver-only metastases in patients with ocular malignant melanoma}

\author{
Svein Dueland $^{1 *}$, Jon Magnus Solheim ${ }^{1,2}$, Aksel Espen Foss ${ }^{2,3}$, Harald Grut ${ }^{1,4}$, Morten Hagness ${ }^{1,2}$ and Pål-Dag Line ${ }^{1-3}$ \\ ${ }^{1}$ Experimental Transplantation and Malignancy Research Group, Division of Surgery, Inflammatory Diseases and Transplantation, Oslo University Hospital, Norway \\ ${ }^{2}$ Section for Transplantation Surgery, Department of Transplantation Medicine, Oslo University Hospital, Norway \\ ${ }^{3}$ Institute of Clinical Medicine, University of Oslo, Norway \\ ${ }^{4}$ Department of Radiology and Nuclear Medicine, Oslo University Hospital, Norway \\ Present address: Department of Radiology, Vestre Viken Hospital Trust, Drammen, Norway
}

\begin{abstract}
Metastases from ocular malignant melanoma spread to the liver in $80-90 \%$ of the cases. The only life prolonging modality in metastatic disease is liver resection, with overall survival ranging from $20-41 \%$ after five years. The aim of the study was to determine if patients with liver only metastases may obtain long survival after liver transplantation. Two patients with ocular malignant melanoma received liver transplantation. Patient 1 had a large tumor load, the largest tumor measuring $160 \mathrm{~mm}$, and multiple smaller lesions, histology showed breach of the liver capsule and occluding thrombosis in the portal vein. Recurrence occurred after 5.6 months and she died of disseminated disease 8.2 months post-transplantation. Patient 2 had a total of 3 lesions, and largest lesion was $15 \mathrm{~mm}$ at histological examination. Liver recurrence occurred at 14.7 months and she died 26.6 months post-transplantation. These two patients with liver only metastatic disease on CT, MRI and PET/CT scan had short disease-free survival, short survival from time of relapse as well as from time of transplantation. The results obtained from this pilot study do not justify further exploration of liver transplantation for patients with ocular malignant melanoma confined to the liver based on tumor size and numbers.
\end{abstract}

ClinicalTrials.gov: NCT01311466

Abbreviations: OMM: ocular malignant melanoma; DFS: diseasefree survival; LT: liver transplantation; MTV: metabolic tumor volume; OS: overall survival

\section{Introduction}

Transplant oncology is an emerging new medical field with more patients with prior or present malignant diseases being considered for solid organ transplantation as well as oncological treatment of malignant diseases in patients with prior organ transplantation. Organ transplantation is considered standard of care internationally for selected patients with primary liver cancer (hepatocellular carcinoma, HCC) with in Milan or "Up-to seven" criteria [1]. Furthermore, selected patients with low grad neuroendocrine tumors are also offered liver transplantation (LT) by several transplant centers [2]. Selected patients with hilar cholangiocarcinoma (Klatskin tumors) receive LT in prospective studies or as standard treatment at some centers [3]. There are also ongoing studies on LT in patients with intra hepatic cholangiocarcinoma (Clinicaltrails.gov NCT 02878473). We have in several publications shown that selected colorectal cancer patients with non-resectable liver only metastases may obtain long overall survival (OS) after LT [4-6]. Solid organ transplant recipients have increased risk of developing several malignant diseases therefore treatment of malignant disease after solid organ transplantation will be of increasing importance. We have previously reported that colorectal cancer patients receiving chemotherapy after LT respond to chemotherapy and in general tolerate chemotherapy combined with immunosuppressive treatment [7].
Ocular malignant melanoma (OMM) is a rare disease, although it is the most common primary intraocular malignancy. The incidence is about 4 per million in the United States and most European countries, while a somewhat higher incidence of 6-9 per million has been reported in the Nordic countries. About 50\% of the patients will develop metastatic disease within 10 years. OMM spreads hematogenously and the liver is the predominant metastatic site in $80 \%-90 \%$ of cases.

The prognosis in patients with metastatic disease is dismal, with an OS of less than 12 months in most chemotherapy studies. OS after trans-arterial chemoembolization treatment, radio-embolization and isolated liver perfusion with melphalan has been reported to result in median OS of 5-21 months, 10-12 months and about 2 years, respectively [8-11].

Ipilimumab (Yervoy ${ }^{\oplus}$ ) is a humanized antibody directed against CTLA- 4 and is approved in the United States by FDA and in Europe by EMEA for treatment of cutaneous malignant melanoma. Ipilimumab has been shown to increase long-term OS in patients with metastatic cutaneous malignant melanoma [12]. However, patients diagnosed

${ }^{*}$ Correspondence to: Svein Dueland MD, Ph.D., Department of Oncology, Oslo University Hospital, Postbox 4950 Nydalen, N-0424 Oslo, Norway, Tel: +47 22 9340 00/ +47930565 48; E-mail: svedue@ous-hf.no

Received: October 08, 2020; Accepted: October 15, 2020; Published: October 19,2020 
with OMM have not been successfully treated by ipilimumab [9] and immune checkpoint inhibitors as ipilimumab and PD-1 inhibitors are not considered standard of care for patients with metastatic OMM. No oncological treatment has documented increased survival in patients with metastatic OMM, so there is an unmet need for better treatment options in these patients with a dismal prognosis when developing metastatic disease.

There are several series on liver resection for metastases from malignant melanoma with surgically accessible metastases. The results from retrospective trials suggest increased survival after liver resection compared to palliative chemotherapy $[13,14]$. However, when liver metastasis occurs, it is most often multiple small deposits not detected by CT scans. Therefore many patients do not receive the scheduled surgical treatment, thus only a small subset of patients may benefit from liver resection [15].

The only previously described LT for OMM was a single case report from 2010. It was an adult-to-adult living donor LT performed on a patient with large tumor load of OMM affecting the portal vein. Recurrence occurred 6 months after surgery and the patient died 8 months after transplantation [16].

The majority of OMM patients have non-resectable disease. Hence, $\mathrm{R} 0$ procedure can only be achieved by liver transplantation. Given the hematogenous spread of OMM, predominantly to the liver and several liver resection series suggesting a prolonged OS after liver resection, a pilot study on LT for liver metastases from OMM was initiated in Oslo, the MECA study (Malignant Melanoma Cancer). Here we report the survival outcome after LT in two patients with liver only metastases from OMM transplanted in this study. Due to the poor outcome, the study was terminated after inclusion of two patients.

\section{Methods}

The study was an open, prospective pilot study (ClinicalTrials.gov Identifier: NCT01311466). The study was approved by Regional Ethics Committee and Institutional Review Board in 2006 on the background of short waiting times and low waitlist mortality for liver transplantation in Norway. Patients with liver metastasis from OMM were referred to Oslo University Hospital. Patients were assessed by a multidisciplinary team consisting of hepatobiliary and transplant surgeons, oncologist and radiologists. After informed consent was obtained, patients with non-resectable disease without signs of extrahepatic disease were evaluated for study inclusion. The main inclusion criteria were complete treatment of the primary site without signs of local recurrence. The treatment of the primary tumor was enucleation and/ or brachytherapy. Extrahepatic malignancy was evaluated by contrast enhanced computed tomography (CT) of chest/abdomen/pelvis and a whole body fluorodeoxyglucose positron emission tomography in combination with CT (PET/CT) within 4 weeks of evaluation at the transplant meeting. Main exclusion criteria were body weight loss of more than $10 \%$ last 6 months, BMI $>30$, standard contraindications for LT and any previous malignancies. At admission for LT, a repeated chest CT was performed to rule out acquired pulmonary metastases on the waiting list; if this CT was negative a perioperative staging laparotomy was carried out with frozen section of lymph nodes from the hepatoduodenal ligament and adjacent tissue. If no signs of extrahepatic malignancy were found LT was performed according to protocol.

Liver metastases from OMM were confirmed histologically by examination of explanted liver. The immunosuppression protocol consisted of sirolimus (mTOR inhibitor), Mycophenolate Mofetil, corticosteroids and induction with Basiliximab and follow-up was as previously described ${ }^{4}$. Relapse of malignant disease was treated individually.

Disease-free survival (DFS) was defined as time from LT to metastatic or local recurrence of OMM. Survival from relapse was calculated as OS minus DFS.

\section{Results}

Two patients with liver only metastases from OMM were included in the study and received LT. Baseline characteristics are given in Table 1. The two patients had very different hepatic tumor load at time of LT. Both patients had received brachytherapy for their primary OMM. The first patient was also treated by enucleation of the affected eye. This patient had also previously received four cycles of trans-arterial chemo embolization (TACE) with drug eluting beads loaded with irinotecan (DEBIRI) before LT. She had progressed on DEBIRI treatment. As common for patients with OMM both patients at time of LT also had multiple small liver lesions not detected by CT-scans. The first patient had large lesions with largest diameter of $15 \mathrm{~cm}$ on CT-scan (Figure $1 \mathrm{~A}$ ) and $16 \mathrm{~cm}$ on histology. The explanted liver was $3.3 \mathrm{~kg}$ in a patient with body weight of $66 \mathrm{~kg}$, representing a liver to body weight of 5 $\%$. The liver metastases from this patient had high uptake on PET/CT (Figure $1 \mathrm{~B}$ ) with a maximum standardized uptake value $\left(\mathrm{SUV}_{\max }\right)$ of 9.16 and a metabolic tumor volume (MTV) of $237 \mathrm{~cm}^{3}$. DFS after LT was 5.6 months. First site of relapse was the liver graft. The metastases were detected by ultrasound and confirmed by cytology. The patient later also developed metastases to lymph nodes, bone, peritoneal carcinomatosis, subcutis and breast. She had rapid deterioration in performance status with hasty growth of liver metastases determined by CT and received no further tumor specific treatment. She died 8.2 months after LT and 2.6 months after the relapse.

The second patient had considerably less advanced metastatic liver disease at time of LT. She had not received any treatment for metastatic
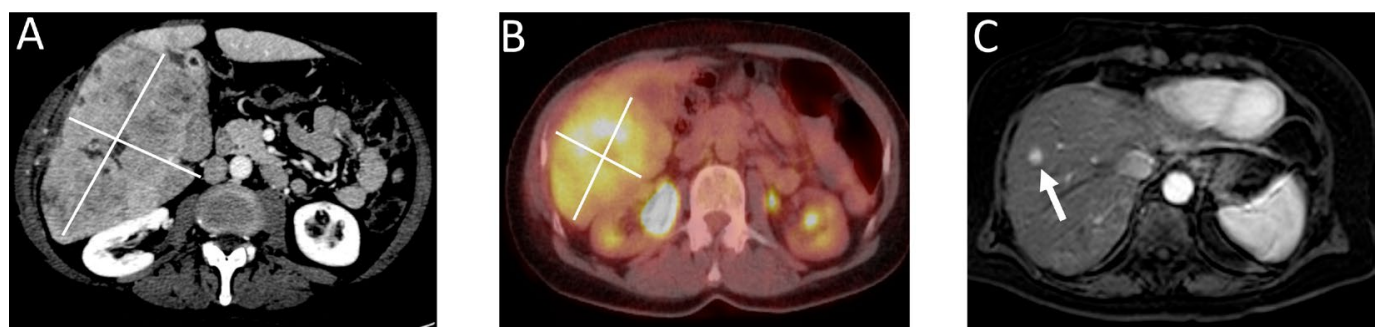

Figure 1A. Contrast enhanced $\mathrm{CT}$ of the first patient with a large metastasis of $15 \mathrm{~cm}$ in the right liver lobe (white lines)

Figure 1B. PET/CT of the first patient who had a large metastasis in the right liver lobe. Metabolic tumor volume was $237 \mathrm{~cm}^{3}$

Figure 1C. Contrast enhanced MRI of the second patient who had 3 small liver metastases. The largest metastasis was only $11 \mathrm{~mm}$ (white arrow) 
Table 1. Baseline characteristics

\begin{tabular}{|l|c|c|}
\hline \multicolumn{1}{|c|}{ Pex } & Female & Patient 2 \\
\hline Age & 61 & Female \\
\hline Primary tumor & Ocular Melanoma & Ocular Melanoma \\
\hline Type & Brachytherapy/enucleation & Brachytherapy \\
\hline Primary Therapy & 31 & 47 \\
\hline $\begin{array}{l}\text { Time from primary diagnosis } \\
\text { to liver metastasis, months }\end{array}$ & 160 & 11 \\
\hline Metastasis liver & Yes & No \\
\hline $\begin{array}{l}\text { Size largest LM, CT/MRI } \\
\text { scan, mm }\end{array}$ & & \\
\hline Capsule breached, histology & & \\
\hline
\end{tabular}

disease before LT. The largest liver metastasis was $11 \mathrm{~mm}$ and number of lesions was 3 evaluated by liver magnetic resonance imaging (MRI) (Figure 1C) and $15 \mathrm{~mm}$ at histological examination. In this patient the liver metastases detected by MRI had no uptake above background on the PET/CT scan. The explanted liver was $1.1 \mathrm{~kg}$ and she had a body weight of $67 \mathrm{~kg}$, representing a liver to body weight ratio of $1.7 \%$. This patient had a DFS of 14.7 months. Relapse was detected in liver by CT, $\mathrm{MRI}$ and PET/CT and confirmed by biopsy. At time of relapse she had metastases only in the liver graft. She had good performance status (ECOG grade 0) and received further treatment with ipilimumab. On this treatment she developed graft rejection as previously described $[17,18]$. Due to graft rejection she received only one infusion of ipilimumab and at evaluation she had progressive disease. She then was offered decarbacine (DTIC) treatment but also progressed on this treatment. This patient had an OS of 26.6 months from LT and 11.9 months from time of relapse.

\section{Discussion}

Metastatic OMM is a disease that is difficult to treat. Many of these patients develop liver metastases as the first and only metastatic site. There are few or no treatment options available that can offer prolonged survival in metastatic disease. In contrast to cutaneous malignant melanoma, immune checkpoint inhibitors have not been effective in OMM [9] and systemic chemotherapy has shown low response rates and OS in OMM patients [9]. Previously experience with TACE with DEBIRI has reported median OS from start of treatment of 9.4 months [8]. In the DEBIRI-study, patients with LDH levels above $250 \mathrm{IU} / 1$ had a median OS of only 3.5 months [8]. The OMM patient with large volume disease included in this study had progressed on DEBIRI and had LD levels above $250 \mathrm{IU} / \mathrm{L}$ both at inclusion in the DEBIRI study and at time of LT. She would probably have had even shorter OS without a LT. The treatment option with longest OS may be isolated liver perfusion with median OS of 22 months [10], however there are considerable side-effects related to this treatment. Randomized control trial with this treatment option is currently ongoing (Clinicaltrials.gov NCT 01785316).

Liver resection for OMM metastasis has shown 5-year OS [17], with up to $41 \%$ in some smaller studies [13]. However, only a minority of the patients are candidates for liver resection due to the number and location of lesions. Furthermore, many of the patients having a laparotomy are considered non-resectable at time of surgery due to detection of many small metastatic liver lesions not identified on pre-operative imaging. Small metastatic lesions in addition to those identified by CT/MRI were also detected at time of LT in the two patients in the current trial and liver resection was therefore not an option.
Others have reported short OS (8 months) after LT in a patient with OMM and extensive disease at time of LT [16]. The two OMM patients included in present study had very different hepatic tumor burden at time of LT. The patient with the smallest tumor load had the longest OS after LT although this was limited to only 27 months, despite the fact that there was no liver uptake on PET and the liver lesions were small ( $11 \mathrm{~mm}$ on MRI-scan). In contrast, colorectal cancer patients with nonresectable liver only disease and low metabolic tumor volume or no liver uptake on PET have long OS (median OS of more than 5 years) after LT $[6,18]$.

HCC patients with tumor within the Milan criteria have 5 years OS of about $75 \%$. However, when these patients have a relapse after LT, median OS from time of relapse is about 8 months [19]. Similar to HCC patients with a relapse after LT, these two OMM patients both had a short OS from time of relapse (less than 12 months), both patients had relapse in the liver graft with no effective systemic treatment available after relapse. Immune checkpoint inhibitors have not shown a substantial increase in OS in OMM patients and immune checkpoint inhibitor administration to solid organ transplant recipients have caused graft rejection/graft loss in some patients [7,20].

The present results from these two OMM patients suggest that it is difficult to use tumor load (size and number of metastases) or PET uptake in liver to select patients for LT. The lack of robust selection criteria and no effective treatment after relapse, indicate that patients with liver metastases from OMM will not reach acceptable OS levels after liver transplantation. Due to the scarcity of liver donor grafts, patients with OMM should therefore not be considered for LT.

\section{Acknowledgements}

This work was supported by South-Eastern Norway Regional Health Authority, Oslo University Hospital and Norwegian Cancer Society.

\section{Disclosure}

The authors declare no conflict of interest.

\section{References}

1. Mazzaferro V, Regalia E, Doci R, Andreola S, Pulvirenti A, et al. (1996) Liver transplantation for the treatment of small hepatocellular carcinomas in patients with cirrhosis. N Engl J Med 334: 693-699. [Crossref]

2. Le Treut YP, Gregoire E, Klempnauer J, Belghiti J, Jouve E, et al. (2013) Liver transplantation for neuroendocrine tumors in Europe-results and trends in patient selection: a 213-case European liver transplant registry study. Ann Surg 257 :807-815. [Crossref]

3. Rosen CB, Heimbach JK, Gores GJ (2010) Liver transplantation for cholangiocarcinoma. Transpl Int 23: 692-697. [Crossref]

4. Hagness M, Foss A, Line PD, Scholz T, Jørgensen PF, et al. (2013) Liver transplantation for nonresectable liver metastases from colorectal cancer. Ann Surg 257: 800-806. [Crossref]

5. Dueland S, Syversveen T, Solheim JM, Solberg S, Grut H, et al. (2020) Survival following liver transplantation for patients with non-resectable liver only colorectal metastases. Ann Surg 271: 530-537. [Crossref]

6. Dueland S, Grut H, Syversveen T, Hagness M, Line PD, et al. (2020) Selection criteria related to long-term survival following liver transplantation for colorectal liver metastasis. Am J Transplant 20: 530-537. [Crossref]

7. Smedman TM, Guren TK, Line PD, Dueland S (2019) Transplant oncology: assessment of response and tolerance to systemic chemotherapy for metastatic colorectal cancer after liver transplantation - a retrospective study. Transpl Int 32: 1144-1150. [Crossref]

8. Carling U, Dorenberg EJ, Haugvik SP, Eide NA, Berntzen DT, et al. (2015) Transarteria Chemoembolization of Liver Metastases from Uveal Melanoma Using IrinotecanLoaded Beads: Treatment Response and Complications. Cardiovasc Intervent Radiol 38: 1532-1541. [Crossref] 
9. Yang J, Manson DK, Marr BP, Carvajal RD (2018) Treatment of uveal melanoma: where are we now? Ther Adv Med Oncol 10: 1758834018757175. [Crossref]

10. Ben-Shabat I, Belgrano V, Ny L, Nilsson J, Lindnér P, et al. (2016) Long-Term FollowUp Evaluation of 68 Patients with Uveal Melanoma Liver Metastases Treated with Isolated Hepatic Perfusion. Ann Surg Oncol 23: 1327-1334. [Crossref]

11. Eschelman DJ, Gonsalves CF, Sato T (2013) Transhepatic therapies for metastatic uveal melanoma. Semin Intervent Radiol 30: 39-48. [Crossref]

12. Batus M, Waheed S, Ruby C, Petersen L, Bines SD, et al. (2013) Optimal management of metastatic melanoma: current strategies and future directions. Am J Clin Dermatol 14: 179-194. [Crossref]

13. Gomez D, Wetherill C, Cheong J, Jones L, Marshall E, et al. (2014) The Liverpoo uveal melanoma liver metastases pathway: outcome following liver resection. J Surg Oncol 109: 542-547. [Crossref]

14. Doussot A, Nardin C, Takaki H, et al. (2015) Liver resection and ablation for metastatic melanoma: A single center experience. J Surg Oncol 111: 962-968. [Crossref]
15. Pinna AD, Yang T, Mazzaferro V, Litchman TD, D'angelica MI, et al. (2018) Liver Transplantation and Hepatic Resection can Achieve Cure for Hepatocellular Carcinoma. Ann Surg 268: 868-875. [Crossref]

16. Zhao J, Yan LN, Li B (2010) Adult-to-adult living donor liver transplantation for malignant metastatic melanoma to the liver. Hepatobiliary Pancreat Dis Int 9: 329332. [Crossref]

17. Rivoire M, Kodjikian L, Baldo S, et al. (2005) Treatment of liver metastases from uveal melanoma. Ann Surg Oncol 12: 422-428. [Crossref]

18. Grut H, Dueland S, Line PD, Revheim ME (2018) The prognostic value of (18)F-FDG $\mathrm{PET} / \mathrm{CT}$ prior to liver transplantation for nonresectable colorectal liver metastases, Eur J Nucl Med Mol Imaging 45: 218-225. [Crossref]

19. Dueland S, Foss A, Solheim JM, Hagness M, Line PD (2018) Survival following liver transplantation for liver-only colorectal metastases compared with hepatocellular carcinoma. Br J Surg 105: 736-742. [Crossref]

20. Dueland S, Guren TK, Boberg KM, Reims HM, Grzyb K, et al. (2017) Acute liver graft rejection after ipilimumab therapy. Ann Oncol 28: 2619-2620. [Crossref]

Copyright: $(2020$ Dueland S. This is an open-access article distributed under the terms of the Creative Commons Attribution License, which permits unrestricted use, distribution, and reproduction in any medium, provided the original author and source are credited. 